\title{
Complete Characterization of Quantum-Optical Processes
}

\author{
Mirko Lobino, ${ }^{1}$ Dmitry Korystov, ${ }^{1}$ Connor Kupchak, ${ }^{1}$ Eden Figueroa,,${ }^{1}$ Barry C. Sanders, ${ }^{1}$ and A. I. Lvovsky ${ }^{1 *}$ \\ ${ }^{1}$ Institute for Quantum Information Science, University of Calgary, Calgary, Alberta T2N 1N4, Canada ${ }^{*}$
}

\begin{abstract}
The technologies of quantum information and quantum control are rapidly improving, but full exploitation of their capabilities requires complete characterization and assessment of processes that occur within quantum devices. We present a method for characterizing, with arbitrarily high accuracy, any quantum optical process. Our protocol recovers complete knowledge of the process by studying, via homodyne tomography, its effect on a set of coherent states, i.e. classical fields produced by common laser sources. We demonstrate the capability of our protocol by evaluating and experimentally verifying the effect of a test process on squeezed vacuum.
\end{abstract}

PACS numbers:

Construction of a complex machine requires precise characterization of each component's properties. In electronics, this information is obtained from network analyzers, which measure circuit response to simple oscillatory inputs, and reveal the device transfer function. Optical quantum technologies, which can be used to build quantum computers [1], precise metrological systems [2], and unconditionally secure communication [3], have similar characterization requirements. In this context, we are interested in the process associated with a quantum circuit component, i.e. in being able to predict the transformation that an arbitrary quantum state will undergo when subjected to the action of the component.

A quantum process $\mathcal{E}$ can be represented by a completely positive, trace-preserving linear map (superoperator) on the linear space $\mathbb{L}(\mathbb{H})$ of all density matrices over Hilbert space H. It can be expressed as a rank-4 tensor that relates the matrix elements of the output $\mathcal{E}(\hat{\rho})$ and input $\hat{\rho}$ states in some basis:

$$
[\mathcal{E}(\hat{\rho})]_{l k}=\sum_{n m} \mathcal{E}_{l k}^{n m} \rho_{n m},
$$

where summation is from 1 to $\operatorname{dim} \mathbb{H}$.

Characterization of a process (known as quantum process tomography, or QPT) means finding all components of the superoperator tensor. It can be implemented by determining the output state for each of the $(\operatorname{dim} \mathbb{H})^{2}$ elements of a spanning set of $\mathbb{L}(\mathbb{H})$. Such a direct approach to QPT [4] was experimentally realized on one qubit teleportation [5], the Hamiltonian evolution of vibrational states of atoms in an optical lattice [6], on a two-qubit controlled-NOT gate [7, 8] and Bellstate filter [9]. As an alternative, ancilla-assisted QPT exploits an isomorphism between processes and states [10] and has been used to characterize a controlled-NOT gate [11] and a general single qubit gate [12, 13]; see [14] for a comparative review of ancilla-assisted QPT.

Existing QPT suffers from serious shortcomings, including either the requirement of an unwieldy set of input states for direct QPT or a high-dimensional entangled input state for ancilla-assisted QPT; these shortcomings deleteriously affect scalability and restrict accessible systems to very low dimension. In optics, QPT has been applied to processes on one

\footnotetext{
*Electronic address: 1vov@ucalgary.ca
}

and two dual-rail qubits, with post-selection based on photon coincidences projecting the input and output states onto these qubit subspaces. This approach cannot provide complete information about a state or a process because optical losses, imperfect sources, detector dark counts, and other imperfections lead to departure from the qubit subspaces. Post-selected tomography can only estimate the fraction of such phenomena by comparing the coincidence rate and the photon production rate [9].

We introduce a scheme that enables complete characterization of a general quantum-optical process. We use optical homodyne tomography followed by maximum likelihood reconstruction to obtain full information on the process across all photon number sectors and also the coherence between sectors. The state reconstruction algorithm provides an efficient method for compensating losses in homodyne detection [15]. As inputs, we use only coherent states that are readily available from a laser source, so our method can be easily scaled up.

We experimentally test our approach by characterizing a quantum process that consists of a simultaneous absorption and phase shift. The reconstructed superoperator allows us to predict, with a fidelity of over $99 \%$, the effect of the process on a squeezed vacuum.

Our method is based on the fact that any density matrix can be represented as a sum of coherent states' density matrices $[16,17]$. Although such a representation (the GlauberSudarshan $P$ function) may be highly singular, it can be arbitrarily closely approximated with a regular $P$ function. By measuring the process output for many coherent states and exploiting the linearity, we can predict the process output for any arbitrary state.

The Glauber-Sudarshan decomposition of a quantum state $\hat{\rho}$ is given by

$$
\hat{\rho}=2 \int P_{\rho}(\alpha)|\alpha\rangle\langle\alpha| d^{2} \alpha
$$

and where $P_{\rho}(\alpha)$ is the state's $P$ function, $\alpha$ is the coherent state with mean position and momentum observables $(x, p)=$ $(\sqrt{2} \operatorname{Re} \alpha, \sqrt{2} \operatorname{Im} \alpha)$. We use the convention $[\hat{x}, \hat{p}]=i$ and integration is performed over the entire phase space. Therefore, if we know the effect $|\alpha\rangle\langle\alpha| \mapsto \hat{\varrho}(\alpha)=\mathcal{E}(|\alpha\rangle\langle\alpha|)$ of the process on all coherent states, we can predict its effect upon state 
$\hat{\rho}$ :

$$
\mathcal{E}(\hat{\rho})=2 \int P_{\rho}(\alpha) \hat{\varrho}(\alpha) d^{2} \alpha .
$$

An obstacle to direct application of this approach is posed by singular behavior of the function $P_{\rho}(\alpha)$. Indeed, the $P$ function exists only as a generalized function, more singular than the Dirac delta function, when the corresponding quantum state has nonclassical features [18].

This can be overcome by applying a theorem proven by Klauder [19]: for any bounded operator $\hat{\rho}$ there exists an operator $\hat{\rho}_{L}$ with continuous and rapidly decreasing $P$ function arbitrarily close to $\hat{\rho}$ in the trace-class norm. The Klauder approximation is obtained as follows: we assume that the Wigner function of $\hat{\rho}$ belongs to the Schwartz class $\mathcal{S}^{2}$, i.e. is infinitely smooth and rapidly decreasing (which is the case for all physically plausible density matrices). The Fourier transform of the operator's Glauber-Sudarshan function $P_{\rho}(\alpha)$ can be expressed as [18]

$$
\tilde{P}_{\rho}\left(k_{x}, k_{p}\right)=\tilde{W}_{\rho}\left(k_{x}, k_{p}\right) \exp \left(\frac{k_{x}^{2}+k_{p}^{2}}{4}\right),
$$

where $\tilde{W}_{\rho}\left(k_{x}, k_{p}\right)$ is the Fourier transform of the operator's Wigner function. The function defined by Eq. 4 always exists and is infinitely smooth (albeit not necessarily square integrable). We multiply $\tilde{P}_{\rho}\left(k_{x}, k_{p}\right)$ by a regularizing function

$$
G_{L}\left(k_{x}, k_{p}\right)=e^{-\left[f\left(k_{x}-L\right)+f\left(-k_{x}-L\right)+f\left(k_{p}-L\right)+f\left(-k_{p}-L\right)\right]}
$$

with $f(y)=y^{4} \exp \left(-1 / y^{2}\right)$ for $y>0, f(y)=0$ for $y \leq 0$. This regularizing function is equal to 1 in a square domain of side $2 L$ and rapidly drops to zero outside. The product $\tilde{P}_{L, \rho}\left(k_{x}, k_{p}\right)=\tilde{P}_{\rho}\left(k_{x}, k_{p}\right) G\left(k_{x}, k_{p}\right)$ now belongs to the Schwartz class. Applying the inverse Fourier transform, we obtain the new Glauber-Sudarshan function $P_{L, \rho}(\alpha)$, which defines the Klauder approximation $\hat{\rho}_{L}$. By choosing $L$ sufficiently high [20], the operator $\hat{\rho}_{L}$ can be made to approximate $\hat{\rho}$ arbitrarily well (Fig. 4A).

As an example, we applied the Klauder approximation to squeezed vacuum, a nonclassical state characterized by a highly singular $P$ function whose Fourier transform grows exponentially with increasing $k_{x}$ and/or $k_{p}$. We tested our protocol with a state that has a noise reduction in the squeezed quadrature of $-1.58 \mathrm{~dB}$ and excess noise in the orthogonal quadrature of $2.91 \mathrm{~dB}$. The function $\tilde{P}\left(k_{x}, k_{p}\right)$ was calculated from the state's density matrix according to Eq. 4 and subsequently regularized as described above using $L=5.2$. Fig. 1A shows $\tilde{P}_{L}\left(k_{x}, k_{p}\right)$ calculated from our experimental data and Fig. 1B displays its inverse Fourier transform $P_{L}(\alpha)$. In Figs. 1C and 1D we compare the Wigner functions of the original state and the one obtained from the regularized $P$ function. The two states exhibit a quantum fidelity of more than 0.9999 .

Although the above method permits finding the process output for an arbitrary input state, it requires one first to determine the input state's $P$ function. This step can be avoided by calculating the process superoperator in the Fock basis, so
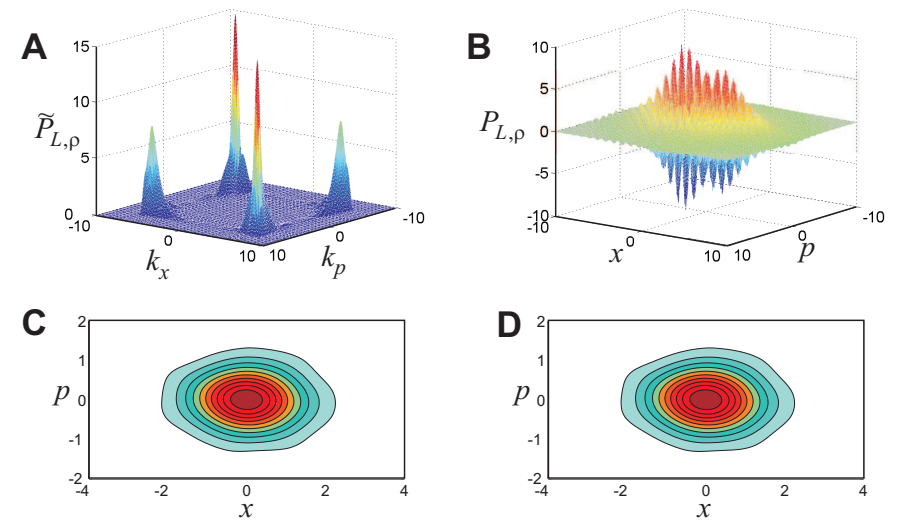

FIG. 1: Regularized Glauber-Sudarshan decomposition of the squeezed state. (A) Absolute value of the regularized Fourier transform of the squeezed vacuum $P$ function. (B) Approximated $P$ function calculated from the inverse Fourier transform of $\tilde{P}_{L, \rho}\left(k_{x}, k_{p}\right)$. (C) and (D) Wigner representations of, respectively, the measured and approximated squeezed vacuum states.

the output can be found from the input density matrix according to Eq. 1. To this end, we express the Glauber-Sudarshan function as

$$
P_{\rho}(\alpha)=\sum_{m n} \rho_{n m} P_{n m}(\alpha)
$$

where $P_{n m}(\alpha)$ is the $P$ function of the operator $|n\rangle\langle m|$. We now replace these functions by their regularized versions $P_{L, n m}(\alpha)$ and rewrite Eq. 3 as

$$
\mathcal{E}(\hat{\rho})=2 \sum_{n m} \rho_{n m} \int P_{L, n m}(\alpha) \hat{\varrho}(\alpha) d^{2} \alpha,
$$

from which we determine the process superoperator as

$$
\mathcal{E}_{l k}^{n m}=2 \int P_{L, n m}(\alpha) \varrho_{l k}(\alpha) d^{2} \alpha .
$$

Prior to applying the latter result to experiments, a number of practical issues have to be addressed. First, parameter $L$ must be chosen to ensure proper approximation of input states. The second issue is that in a realistic experiment, the measurement can be done only for coherent states whose amplitude does not exceed a certain maximum $\alpha_{\max }$. Finally, the experiment can only be performed with a finite, discrete set of coherent states. Density matrix elements $\varrho_{l k}(\alpha)$ for an arbitrary $\alpha$, required for calculating the superoperator, must then be obtained by polynomial interpolation. These matters are discussed in [20].

A simplification arises for phase-symmetric processes, in which there is no phase coherence between the "processing unit" and input states. In this case, if two inputs $\hat{\rho}$ and $\hat{\rho}_{1}$ are different by an optical phase shift $\hat{U}(\varphi)$, the states $\mathcal{E}(\hat{\rho})$ and $\mathcal{E}\left(\hat{\rho}_{1}\right)$ will differ by the same phase shift:

$$
\mathcal{E}\left[\hat{U}(\varphi) \hat{\rho} \hat{U}^{\dagger}(\varphi)\right]=\hat{U}(\varphi) \mathcal{E}(\hat{\rho}) \hat{U}^{\dagger}(\varphi) .
$$


Then, if we know the effect of the process on a coherent state $|\alpha\rangle$, we also know what happens to $\left|\alpha e^{i \varphi}\right\rangle$, so it is enough to perform measurements on input coherent states with real, positive amplitudes. For the process superoperator in the Fock basis, the phase symmetry implies that $\mathcal{E}_{m n}^{k l}$ vanishes unless $k-l=m-n$.

The process studied in our experiment was electro-optical amplitude and phase modulation of the optical field. The process was implemented using an electro optical modulator (EOM) followed by a polarizer. The field experienced minimal distortion when a bias voltage $V_{1}=100 \mathrm{~V}$ was applied to the EOM. Switching the voltage to $V_{2}=50 \mathrm{~V}$ produced birefringence, and thus losses at the polarizer, along with a phase shift.

A continuous-wave Ti:Sapphire laser at $795 \mathrm{~nm}$ was the coherent state source used for the device characterization. We reconstructed the input and output states at 11 different input amplitude levels between $\alpha_{1}=0$ and $\alpha_{11}=10.9$. In order to keep track of the relative phase shift, the EOM voltage was switched between $V_{1}$ and $V_{2}$ every $100 \mu$ s (Fig. 2A, top) while the phase of the local oscillator was linearly scanned by a piezoelectric transducer at $100 \mathrm{~Hz}$. The homodyne photocurrent was recorded with an oscilloscope. To obtain quadrature measurements, the photocurrent was integrated over time intervals of $20 \mathrm{~ns}$. The bottom plot in Fig. 2A shows the recovered quadrature values after normalization to the vacuum noise. The time dependence of the local oscillator phase was recovered from the slow, sinusoidal variation of the average homodyne photocurrent as a function of time.

In this manner, for each input amplitude, we sampled 50,000 phase and quadrature values for both the input and output states and used them to calculate density matrices by likelihood maximization $[15,21]$ (Fig. 2B). The output state reconstruction showed a phase shift of $36^{\circ}$ and a loss of $34 \%$ with respect to the input state.

The interpolated experimental data have been used to determine the process superoperator tensor. We used the phase symmetry assumption in Eq. 9, which is justified by the fact that the EOM driver is independent from the master laser. The elements $\mathcal{E}_{k k}^{m m}$ of the tensor in the photon number basis are plotted in Fig. 3A. This plot should be interpreted as follows: for a given input Fock state $|m\rangle$, the values of $\mathcal{E}_{k k}^{m m}$ give the diagonal elements of the output density matrix. For example, the single-photon state $|1\rangle$ after passing through the EOM will be transformed into a statistical mixture of the single-photon and vacuum states. A theoretical prediction for the process tensor has been calculated using the Bernoulli transformation to account for a lossy channel and a phase shift superoperator; the superoperator diagonal elements in the Fock basis are displayed in Fig. 3B, and these diagonal elements bear close resemblance to the experimental result. A similar agreement was also obtained for non-diagonal terms of the superoperator, but it is not shown here.

For additional verification, we applied this result to predict the effect of the device on the squeezed vacuum state described in the previous section. This state was produced by pumping an optical parametric amplifier (OPA) in bow tie configuration with the second harmonic of the Ti:Sapphire
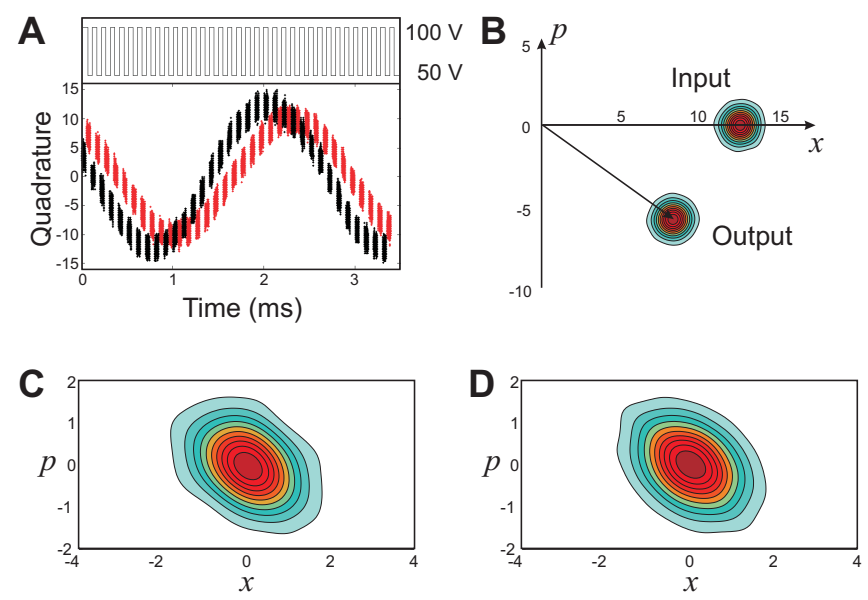

FIG. 2: (A) Time-dependent quadrature values acquired from homodyne detection of a coherent state with input $\alpha=8.3$. Black dots correspond to the state before the process; red dots, after the process. The top curve shows the EOM driving voltage. (B) The Wigner function of the coherent state before and after the process. (C) and (D) Wigner representations of the measured output squeezed state compared to the one obtained from process tomography.
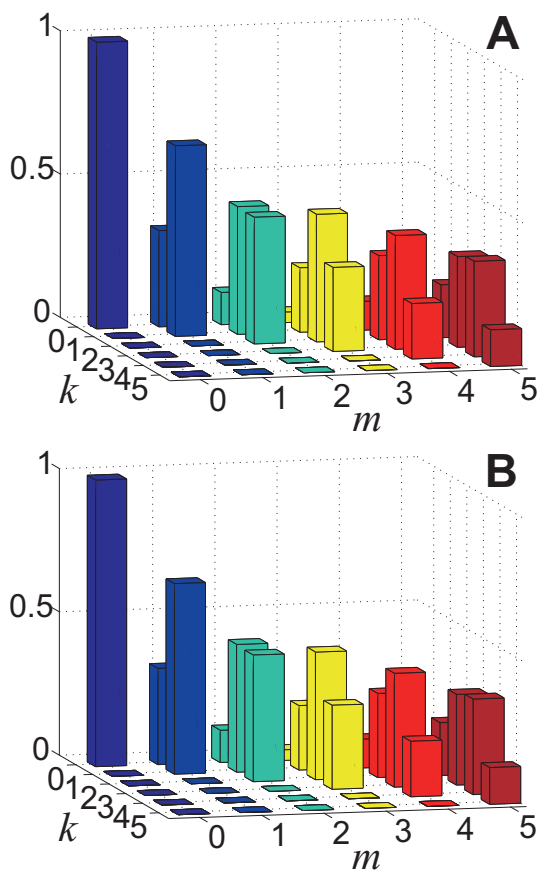

FIG. 3: The "diagonal" values of the superoperator $\mathcal{E}_{k k}^{m m}$. (A): as obtained in the experiment. (B): theoretical model.

laser and using a periodically poled $\mathrm{KTiOPO}_{4}$ crystal as nonlinear medium[22, 23].

The state before (Fig. 1C) and after (Fig. 2C) the process was reconstructed using homodyne tomography as described above. By applying the process superoperator to the input squeezed state, we predict the process output (Fig. 2D). The maximum (minimum) quadrature noise variance amounted 
to $2.19(-1.07) \mathrm{dB}$ for the measured state, and $2.15(-0.95)$ $\mathrm{dB}$ for the prediction, corresponding to a quantum fidelity of $0.9935 \pm 0.0002$.

Whereas here we demonstrate our tomographic method for single-mode inputs, multimode or multichannel processes can be characterized using multimode $P$ representation, multiple homodyne detectors and feeding product coherent states as inputs. Theoretical and experimental analysis of the multimode setting will be discussed elsewhere.

Our method overcomes significant limitations of previous optical QPT schemes. Process characterization is not restricted to a Hilbert space associated with a specific qubit, and thus reveals the imperfections of a quantum information processing unit. Additionally, it uses only coherent states as inputs, which are readily available from the laser and whose intensities and phases are easily manipulated. This permits characterization of complex processes employed in quantum information processing and communication.

Supporting Material. In order to compute the $\mathrm{P}$ function $P_{L, \rho}(\alpha)$ by the Klauder approximation [19] and the superoperator terms from

$$
\mathcal{E}_{l k}^{n m}=2 \int P_{L, n m}(\alpha) \varrho_{l k}(\alpha) d^{2} \alpha,
$$

where $P_{L, n m}(\alpha)$ is the regularized $\mathrm{P}$ function of the operator $|n\rangle\langle m|$, the values of two parameters must be set: the size $L$ of the Fourier domain, and the maximum coherent state amplitude $\alpha_{\max }$. As evidenced by Fig. 4A, no value of $L$ can ensure universally high fidelity for all optical states. However, for many practical applications it is reasonable to restrict the input states to subspace $\mathrm{H}_{N}=\operatorname{span}(|0\rangle, \ldots,|N\rangle)$ with a limited number of photons. Under this restriction, we can seek the lowest $L$ that, in the worst case over all density matrices in $\mathrm{H}_{N}$, yields an approximation to the input state with required fidelity $F$.

We employed the genetic optimization algorithm [24] to calculate the required value of $L$ for several low values of $N$ and ascertained that the worst case scenario corresponds to the Fock state $|N\rangle$. This is not surprising, because the $P$ function of this state contains the highest order derivative of the Dirac delta in $\mathrm{H}_{N}$. We assume this rule to hold for arbitrary $N$.

Restricting the amplitude of coherent states for which the measurements are performed effectively introduces finite integration limits in Eq. 1, entailing additional fidelity loss. Fig. 4B shows the lowest $\alpha_{\max }$ required for approximating the $N$-photon Fock state (which, again, appears to be the worst case in $\mathrm{H}_{N}$ ) with a 99 per cent fidelity. Notably, this quantity is much larger than that expected from the behavior of the corresponding Wigner function. This is because the regularized $P$ function exhibits strong oscillations in a much wider phase space region than does the Wigner function.

Even with a finite $\alpha_{\max }$, reconstructing the superoperator requires knowing the process output for a continuum of coherent states. In an experiment, we choose a finite set of inputs $\left|\alpha_{i}\right\rangle$ from a laser source with varying, but known, amplitudes and phases, and perform homodyne tomography to determine the corresponding output density matrices $\varrho_{l k}\left(\alpha_{i}\right)$ in the Fock basis. For an arbitrary $\alpha$, density matrix elements $\varrho_{l k}(\alpha)$ are then obtained by polynomial interpolation.

The degree of the polynomial over $\alpha$ can be reduced for Gaussian and near-Gaussian maps, which comprise all linearoptical processes and $\chi^{(2)}$ nonlinearities. For each measured output $\hat{\varrho}\left(\alpha_{i}\right)$ we infer its mean complex amplitude $\langle\hat{a}\rangle=$ $\operatorname{Tr}\left(\hat{a} \varrho\left(\alpha_{i}\right)\right)$. Then we apply the displacement operator

$$
\varrho_{i}^{\langle\hat{a}\rangle}=D(-\langle\hat{a}\rangle) \varrho\left(\alpha_{i}\right) D^{\dagger}(-\langle\hat{a}\rangle) .
$$

to center the Wigner function at the origin of the phase space. The matrix elements $\varrho_{l k}^{\langle\hat{a}\rangle}$, as well as the mean amplitude $\langle\hat{a}\rangle$, can now be fitted with a low-degree polynomial over $\alpha$, allowing for a highly efficient reconstruction process. Good performance of this algorithm for Gaussian preserving maps is explained by the fact that Gaussian states are entirely characterized by the first and second centered moments $\overline{(x-\sqrt{2} \operatorname{Re}\langle\hat{a}\rangle)^{m}(p-\sqrt{2} \operatorname{Im}\langle\hat{a}\rangle)^{n}}$ of their Wigner distribution.
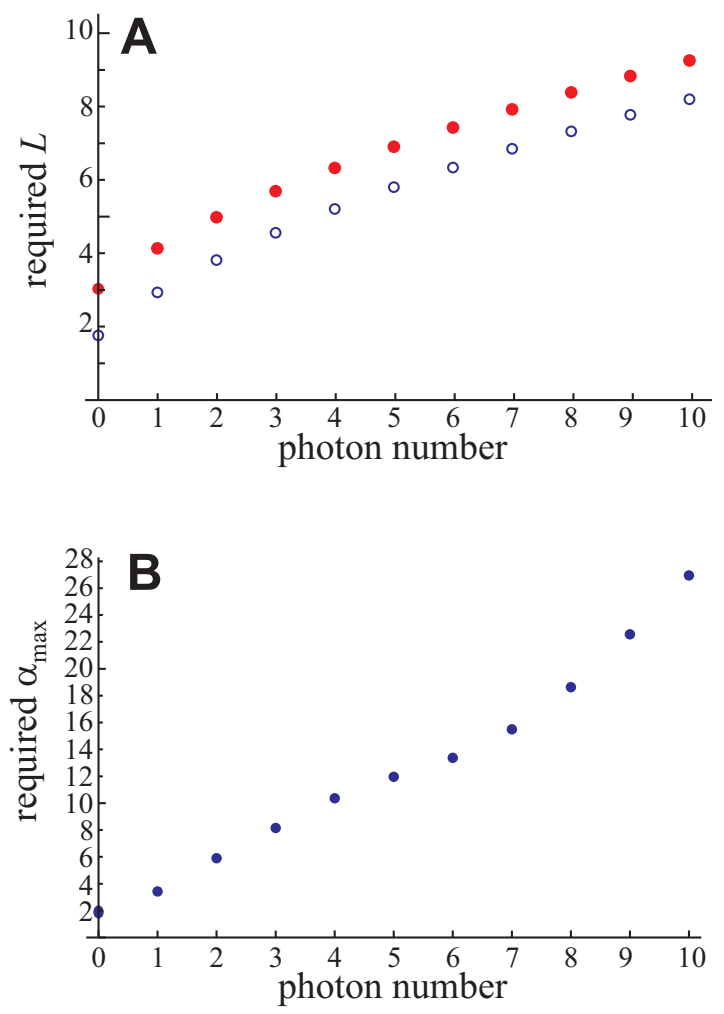

FIG. 4: Requirements for faithful approximation of photon number states with a regularized, restricted Glauber-Sudarshan decomposition. (A): the low pass filtering parameter $L$ needed to achieve a 99.99-\% fidelity ( $\bullet$ ) and 99-\% fidelity (०). (B): the lowest $\alpha_{\max }$ required for a $99-\%$ fidelity. 
[1] E. Knill, R. Laflamme, G. J. Milburn, Nature 409, 46 (2000).

[2] J. Ye, H. J. Kimble, H. Katori, Science 320, 1734(2008).

[3] N. Gisin, G. Ribordy, W. Tittel, H. Zbinden, Rev. Mod. Phys. 74, 145 (2002).

[4] J. F. Poyatos, J. I. Cirac, P. Zoller, Phys. Rev. Lett. 78, 390 (1997).

[5] M. A. Nielsen, E. Knill, R. Laflamme, Nature 396, 52 (1998).

[6] S. H. Myrskog, J. K. Fox, M. W. Mitchell, A. M. Steinberg, Phys. Rev. A 72, 013615 (2005).

[7] J. L. O'Brien et al., Phys. Rev. Lett. 93, 080502 (2004).

[8] A. M. Childs, I. L. Chuang, D. W. Lueng Phys. Rev. A 64, 012314 (2001).

[9] M. W. Mitchell, C. W. Ellenor, S. Schneider, A. M. Steinberg Phys. Rev. Lett. 91, 120402 (2003).

[10] G. M. D'Ariano, P. Lo Presti, Phys. Rev. Lett. 86, 4195 (2001).

[11] M. Riebe et al., Phys. Rev. Lett. 97, 220407 (2006).

[12] J. B. Altepeter et al., Phys. Rev. Lett. 90, 193601 (2003).

[13] F. De Martini, A. Mazzei, M. Ricci, G. M. D’Ariano, Phys. Rev. A 67, 062307 (2003).

[14] M. Mohseni, A. T. Rezakhani, D. A. Lidar, Phys. Rev. A 77,
032322 (2008).

[15] A. I. Lvovsky, J. Opt. B: Q. Semiclass. Opt. 6, S556 (2004).

[16] R. J. Glauber, Phys. Rev. Lett. 10, 84 (1963).

[17] E. C. G. Sudarshan, Phys. Rev. Lett. 10, 227 (1963).

[18] U. Leonhardt, Measuring the Quantum State of Light (Cambridge University Press, Cambridge, 1997).

[19] J. R. Klauder, Phys. Rev. Lett. 16, 534 (1966).

[20] Supporting Online Material.

[21] J. Řeháček, Z. Hradil, E. Knill, A. I. Lvovsky, Phys. Rev. A 75 , 042108 (2007).

[22] J. Appel, D. Hoffman, E. Figueroa, A. I. Lvovsky, Phys. Rev. A 75, 035802 (2007).

[23] J. Appel, E. Figueroa, D. Korystov, M. Lobino, A. I. Lvovsky, Phys. Rev. Lett. 100, 093602 (2008).

[24] Lance Chambers, Practical Handbook of Genetic Algorithms: Applications Vol.I (CRC Press Inc, Boca Raton 1995).

[25] This work was supported by NSERC, CIFAR, iCORE, AIF, CFI and QuantumWorks. A. I. L. is a CIFAR scholar, B. C. S. is a CIFAR associate. 